Transformative Research Award initiative), Principle Investigator, April 1st. 2019 - present. K. Ohkawa: 1; C; 17H02080, Japan Society for the Promotion of Science Ohkawa, Kousaku (PI) 04/01/17-03/31/21. F. Hsu: 1; C; Institute for Clinical and Translational Science (ICTS) Pilot Award (NIH Director's Transformative Research Award initiative). S. Suzuki: 1; C; Institute for Clinical and Translational Science (ICTS) Pilot Award (NIH Director's Transformative Research Award initiative).

\section{P-037 DEVELOPMENT OF A PROTEOMIC BIOMARKER SIGNATURE FOR IDENTIFYING INTRACRANIAL ANEURYSMS}

D Nistal ${ }^{*}$, M Martini, T Hardigan, R Song, J Mocco, C Kellner. Neurosurgery, Icahn School of Medicine, New York, NY

\subsection{6/neurintsurg-2019-SNIS.73}

Introduction Intracranial aneurysms occur in approximately 1$2 \%$ of the general population and are the leading cause for subarachnoid hemorrhage (SAH). Recent studies have shown the utility of using multiplex immunoassays in biomarker discovery for a variety of diseases. Although a number of biomarkers have been identified to be related to intracranial aneurysms, a proteomic signature has not been defined to predict the presence of an intracranial aneurysm. In this study, we utilized proteomic data from patients with known intracranial aneurysms and healthy controls to identify a proteomic signature in the serum to predict the presence of an aneurysm.

Methods Fifty-six patients were prospectively enrolled in this study, 28 of which with unruptured intracranial aneurysms and 28 sex, age, and comorbidity matched healthy controls. Serum was collected from each patient. Protein expression levels were determined using the Proseek multiplex immunoassay (http://www.olink.com/), which included 92 known inflammatory markers. Univariate and multivariate logistic regression models were constructed and systematically assessed using statistical and biological inferences to identify phenotypic and proteomic variables that significantly predicted presence of an aneurysm.

Results Of the 28 patients with intracranial aneurysms, $82.1 \%$ $(n=23)$ were female, $46.4 \% \quad(n=13)$ were never smokers, $35.7 \%(n=10)$ were former smokers, and $17.9 \%(n=5)$ were current smokers. Healthy controls were matched on a 1:1 basis, with age \pm 5 years. In the patient cohort the mean aneurysm size was $8.9 \mathrm{~mm}$ with the most common location being anterior communicating artery $(35.7 \%, n=10)$. Upon univariate analysis, increased expression of 10 analytes were found to be independent predictors of the presence of an intracranial aneurysm. At the univariate level, IL17 $(\mathrm{OR}=12.01 ; 95 \%$ CI $4.16-$ 53.17; $\mathrm{p}<0.00001)$ and $\mathrm{CD} 244(\mathrm{OR}=34.19 ; 95 \%$ CI 6.14 $344.81 ; \mathrm{p}=0.000531)$ were found to be the most predictive. At the multivariate level, while controlling for age, sex, hypertension, and smoking status, IL17 remained a significant predictor of the presence of an intracranial aneurysm $(\mathrm{OR}=32.70$, 95\% CI 4.64 - 778.81; $\mathrm{p}=0.00485)$.

Conclusion This study leveraged individualized data from patients with intracranial aneurysms to determine which proteomic variables predict the presence of an intracranial aneurysm while controlling for essential clinical covariates. Larger multi-omic studies are warranted to develop a more comprehensive biomarker signature for intracranial aneurysms.
Disclosures D. Nistal: None. M. Martini: None. T. Hardigan: None. R. Song: None. J. Mocco: 1; C; Penumbra Inc.. 2; C; Cerebrotech, Rebound Therapeutics, TSP Inc, Lazarus Effect, Medina, Pulse Vascular. C. Kellner: 1; C; Siemens, Penumbra, Bee Foundation.

\section{P-038 PREDICTORS OF VENTRICULOSTOMY INFECTION IN A LARGE COHORT}

A Sweid*, S Tjoumakaris, C Wamsley, E Mann, C Neely, J Head, M Gooch, N Herial, T Alexander, S Missios, N Chalouhi, R Rosenwasser, P Jabbour. Neurosurgery, Thomas Jefferson University, Philadelphia, PA

10.1136/neurintsurg-2019-SNIS.74

Background External ventricular drain (EVD) placement is a common neurosurgical procedure. While this procedure is simple and effective, infection is a major limiting factor. Factors predictive of infection reported in the literature are not conclusive.

Objective The aim of this retrospective single-center large sample was to assess the rate and factors predictive of infection.

Methods We performed a retrospective review of patients who underwent EVD placement between January 2012 and January 2018.

Results A total of 714 patients were included in the study. The infection rate was $3.6 \%(26 / 714)$. In $45 \%$ of the cases, the indication for ventriculostomy placement was for acute subarachnoid hemorrhage (aSAH) or intracerebral hemorrhage (ICH). Of the factors that were an independently significant predictor of ventriculostomy-associated infection (VAI) was the placement of a second EVD; the likelihood was eight times higher than if only a single EVD was placed (OR: 7.95, 95\% CI: 1.01-62.9, p<0.05). Another factor was routine CSF sampling, associated with greater than nine times higher likelihood of VAI compared to no routine CSF sampling (OR: 9.20, 95\% CI: 1.93-43.9, p<0.01). Intrathecal (IT) drug deliveries were also associated with a higher likelihood of VAI (OR: 55.7, 95\%CI: 2.92-

1060, $\mathrm{p}<0.01)$. Non-significant factors were day vs. night, location (ICU/ER vs. OR), duration, CSF leak, suture type, system flushing.

Conclusion Our study shows that the rate of VAI was $3.6 \%$. Only three variables were significant independent predictors of VAI; these were the replacement of the EVD, routine CSF aspiration, and intrathecal drug delivery.

Disclosures A. Sweid: None. S. Tjoumakaris: None. C. Wamsley: None. E. Mann: None. C. Neely: None. J. Head: None. M. Gooch: None. N. Herial: None. T. Alexander: None. S. Missios: None. N. Chalouhi: None. R. Rosenwasser: None. P. Jabbour: None.

\section{P-039 INTRAVASCULAR ULTRASOUND OF FLOW DIVERTERS IN ANEURYSM TREATMENT}

${ }^{1} \mathrm{~A}$ Nicholson*, 'S Taylor, ${ }^{2} \mathrm{C}$ Woods, ${ }^{1} \mathrm{~J}$ Cuoco, ${ }^{1} \mathrm{~B}$ Klein, ${ }^{1} \mathrm{E}$ Guilliams, ${ }^{1} \mathrm{E}$ Marvin, IJ Entwistle. ${ }^{1}$ Neurosurgery, Carilion Clinic, Roanoke, VA; ${ }^{2}$ Virginia Tech Carilion School of Medicine, Roanoke, VA

10.1136/neurintsurg-2019-SNIS.75

Introduction/Purpose The use of flow diverting stents for the treatment of cerebral aneurysms has significantly increased since the PUFS (Pipeline for Uncoilable of Failed Aneurysms) 
trial was released in 2013. Based on the extensive literature on flow diverting stents one of the most critical features of stent placement is vessel wall apposition. Examining the cardiology literature, stents placed using intravascular ultrasound (IVUS) show a 50\% decreased in-hospital mortality and a $34 \%$ decrease in major adverse cardiac events in the 24 months following stent placement. Although the degree of flow diversion and wall apposition in cerebral aneurysm treatments can be assessed on angiography, it often provides an incomplete picture, which can result in the unnecessary placement of multiple overlapping stents. In this study, we assess the utility and feasibility of intravascular ultrasound (IVUS) in determining wall apposition and quantifying flow diversion post stent placement.

Materials and methods Five (5) consecutive patients were selected who underwent angiography with possible flow diverting stent placement for known unruptured cerebral aneurysms at our institution. All five patients underwent full diagnostic angiography, followed by a separate endovascular surgery using flow diverting stents. Prior to stent placement, a Visions PV 0.014P RX (Philips) was introduced through the intermediate catheter past the aneurysm and a pullback recording was performed using Chromaflow for analysis. A Pipeline Flex Embolization Device (Medtronic) was then placed in the standard fashion. After stent placement, the IVUS catheter was re-introduced and another pullback recording obtained. Pre- and post- stent vessel diameter, as well as wall apposition was assessed. Flow diversion was assessed using Chromaflow power Doppler measurements.

Results Intravascular ultrasound was safe and technically feasible in $100 \%(5 / 5)$ of the patients analyzed. Vessel wall dimensions were compared to angiography in these patients, and the IVUS measurements influenced a change in stent size in $60 \%$ $(3 / 5)$ patients. Likewise, Doppler flow measurements influenced the decision to not place additional stents in $60 \%$ of patients. Doppler flow decreased in the aneurysms by $60-$ $100 \%$ after flow diversion, and $90-100 \%$ at the conclusion of the procedure. Follow up angiography at 3- and/or 6-months post stenting was obtained in 4 of our patients.

Conclusion Intravascular ultrasound is a safe and effective tool in the evaluation and treatment of cerebral aneurysms using flow diverting stents. In our small study our use of this technology improved accuracy of device sizing, wall apposition, and decreases the number of stents need to treat aneurysms.

Disclosures A. Nicholson: None. S. Taylor: None. C. Woods: None. J. Cuoco: None. B. Klein: None. E. Guilliams: None. E. Marvin: None. J. Entwistle: None.

\section{Electronic poster abstracts}

\section{E-001 TRAUMATIC ARTERIOVENOUS FISTULA OF THE MIDDLE MENINGEAL ARTERY MIMICKING A CAVERNOUS SINUS FISTULA DURING INFANCY}

${ }^{1,2} \mathrm{R}$ Dahl ${ }^{*},{ }^{3} \mathrm{G}$ Roende, ${ }^{4} \mathrm{~A}$ Born, ${ }^{1} \mathrm{G}$ Benndorf. ${ }^{1}$ Department of Radiology, University Hospital Rigshospitalet, Copenhagen, Denmark; ${ }^{2}$ Department of Abdominal Surgery, University Hospital Aalborg, Aalborg, Denmark; ${ }^{3}$ Department of Pediatrics, Copenhagen University Hospital Hvidovre, Hvidovre, Denmark; ${ }^{4}$ Department of Pediatrics, University Hospital Rigshospitalet, Copenhagen, Denmark

\subsection{6/neurintsurg-2019-SNIS.76}

Background Traumatic arteriovenous fistulas (AVFs) involving the middle meningeal artery (MMA) mimicking a cavernous sinus fistula (CSF) are rare lesions that may develop after arterial injury to the MMA in proximity to a meningeal vein or dural sinus. The authors present the unusual case of an infant who sustained a minor head trauma after a short vertical fall. Clinical history A 6-month-old girl initially presented with a bruit over the left temple. Symptoms progressed with increasing proptosis and enlarged conjunctival vessels on her left eye. Due to lacking clear evidence of a sufficient trauma in her history, an orbital tumor or vascular malformation were

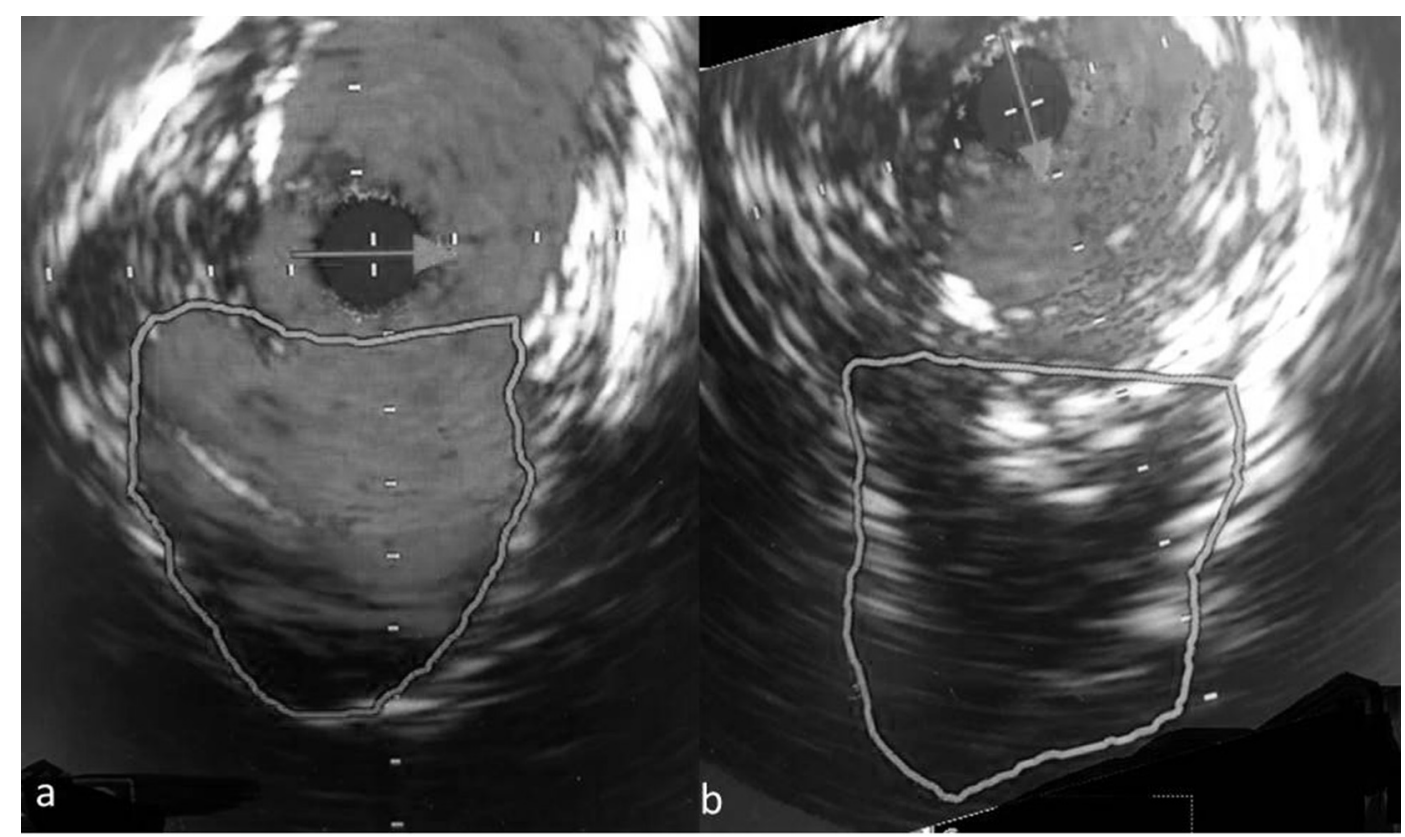

Abstract P-039 Figure 1 Intravascular ultrasound (IVUS) with Chromaflow power doppler assessment of a posterior communicating artery aneurysm [outlined in green] pre-(a) and post- (b) flow-diverting stent placement. 